\title{
Optimal Control Systems with Constrains Defined on Unbounded Sets
}

\author{
Dorota Bors, Marek Majewski and Stanisław Walczak \\ University of Łódź
}

Poland*

\section{Introduction}

We consider a quasilinear control system of the form

$$
\begin{aligned}
-z^{\prime \prime}(t)+A(t, u(t)) z(t)+\alpha \varphi(t, z(t)) & =B(t) u(t), \\
z(0) & =0,
\end{aligned}
$$

with the integral constrain of inequality type:

$$
\int_{I} \phi(t, z(t)) d t \leq l
$$

and the integral quality indicator:

$$
F(z, u):=\int_{I} f(z(t), u(t), t) d t \rightarrow \min ,
$$

where $t \in I:=[0, \infty), \alpha \in \mathbb{R}, z(\cdot) \in H_{0}^{1}\left(I, \mathbb{R}^{N}\right), u(\cdot) \in L^{\infty}\left(I, \mathbb{R}^{M}\right), A(\cdot, \cdot) \in L^{\infty}\left(I \times U, \mathbb{R}^{N \times N}\right)$, $B(\cdot) \in L^{1}\left(I, \mathbb{R}^{N \times M}\right) \cap L^{2}\left(I, \mathbb{R}^{N \times M}\right), \varphi(\cdot, \cdot) \in C\left(I \times \mathbb{R}^{N}, \mathbb{R}\right), \phi(\cdot, \cdot) \in C\left(I \times \mathbb{R}^{N}, \mathbb{R}\right), \phi(t, \cdot)$ is differentiable and $\varphi(t, \cdot)=\nabla \phi(t, \cdot)$, for $t \in I, U \subset \mathbb{R}^{N}, N, M \geq 1$ and $f: \mathbb{R}^{N} \times \mathbb{R}^{M} \times I \rightarrow \mathbb{R}$.

We define a space $H_{0}^{1}\left(I, \mathbb{R}^{N}\right)$ of trajectories as a set of functions which are absolutely continuous on any compact subinterval $I_{0} \subset I$ and satisfy conditions:

$$
z(0)=0, \quad \int_{I}|z(t)|^{2} d t<\infty \text { and } \int_{I}\left|z^{\prime}(t)\right|^{2} d t<\infty,
$$

i.e. $z(0)=0$ and $z(\cdot), z^{\prime}(\cdot) \in L^{2}\left(I, \mathbb{R}^{N}\right)$.

Let us recall that the necessary and sufficient condition for a function $z:[a, b] \rightarrow \mathbb{R}$ to be absolutely continuous is the following integral representation

$$
z(t)=\int_{a}^{t} v(t) d t+c \quad \text { for } t \in[a, b],
$$

where $v \in L^{1}([a, b], \mathbb{R}), c \in \mathbb{R}$ Łojasiewicz (1974). A function $z:[a, b] \rightarrow \mathbb{R}^{N}$ is absolutely continuous if each coordinate function $z_{i}(\cdot)$ is absolutely continuous. Furthermore, for almost

*This work is a part of the research project N514 027 32/3630 supported by the Ministry of Science and Higher Education (Poland). 
every $t \in[a, b]$ an absolutely continuous function $z:[a, b] \rightarrow \mathbb{R}^{N}$ possesses finite and integrable derivative $z^{\prime}(\cdot)$ (see Łojasiewicz (1974)) Moreover,

$$
z^{\prime}(t)=v(t) \quad \text { for a.e. } t \in[a, b]
$$

where

$$
z(t)=\int_{a}^{t} v(t) d t+c .
$$

(see Łojasiewicz (1974) for more details). The space of trajectories $H_{0}^{1}\left(I, \mathbb{R}^{N}\right)$ defined above is a Banach space with the norm

$$
\|z\|^{2}:=\int_{I}\left(|z(t)|^{2}+\left|z^{\prime}(t)\right|^{2}\right) d t
$$

One can prove that for a function $z(\cdot) \in H_{0}^{1}\left(I, \mathbb{R}^{N}\right)$ we have

$$
z(\infty):=\lim _{t \rightarrow \infty} z(t)=0 .
$$

Moreover $H_{0}^{1}\left(I, \mathbb{R}^{N}\right)$ is a Hilbert space with the scalar product

$$
\langle z, v\rangle:=\int_{I}\left(\langle z(t), v(t)\rangle+\left\langle z^{\prime}(t), v^{\prime}(t)\right\rangle\right) d t
$$

see for example Adams (1975), Kufner A (et al.) and references therein.

As a set of admissible controls we take

$$
\mathcal{U}:=\left\{u(\cdot) \in L^{\infty}\left(I, \mathbb{R}^{M}\right):|u(t)| \leq v_{0}(t) \wedge u(t) \in U \text { for a.e. } t \in I\right\},
$$

where $v_{0}(\cdot) \in L^{2}(I, \mathbb{R}), U \subset \mathbb{R}^{M}$. The fundamental difference between above optimal control problem (1)-(5) and a classical optimal control problem is that control system (1) and functional (3) are defined on an unbounded time interval.

Differential systems of the form (1) are often referred to as the Newton's systems with an infinite time horizon. By inequalities (4), i.e. $\int_{I}|z(t)|^{2} d t<\infty$ and $\int_{I}\left|z^{\prime}(t)\right|^{2} d t<\infty$ and some natural assumptions it may be concluded that the global kinetic energy

$$
E_{k}=\int_{0}^{\infty}\left|z^{\prime}(t)\right|^{2} d t
$$

and the potential energy

$$
E_{p}=\int_{0}^{\infty}\langle A(t, u(t)) z(t)+\alpha \phi(t, z(t))-B(t) u(t), z(t)\rangle d t
$$

are finite. In the real world, we meet only such a kind of the dynamical systems (see for example Lieb \& Loss (2001)). 


\section{Existence of solutions}

In this chapter we prove that the problem (1)-(2) possesses solutions for each control $u_{0}(\cdot) \in \mathcal{U}$. We say that for a given $u(\cdot) \in \mathcal{U}$ a function $z_{u}(\cdot) \in H_{0}^{1}\left(I, \mathbb{R}^{N}\right)$ solves (1)-(2) if there is a constant $\alpha_{0} \geq 0$ that

$$
\begin{aligned}
-z_{u}^{\prime \prime}(t)+A(t, u(t)) z_{u}(t)+\alpha_{0} \varphi\left(t, z_{u}(t)\right) & =B(t) u(t) \quad \text { for a.e. } t \in I, \\
z_{u}(0) & =0, \\
\int_{I} \phi\left(t, z_{u}(t)\right) d t & \leq l .
\end{aligned}
$$

The above type of solution is called a Caratheodory solution.

We will make the following assumptions:

(A1) the matrix $A(t, u)$ is positively defined and symmetric for each $t \in I$ and each $u \in U$,

(A2) the function $\phi(t, \cdot)$ is strictly convex and there are a constant $a_{1}>0$ and a function $a_{2}(\cdot) \in L^{1}(I, \mathbb{R})$, such that

for $t \in I$ and $z \in \mathbb{R}^{N}$,

$$
|\phi(t, z)| \leq a_{1}|z|^{2}+a_{2}(t)
$$

(A3) there is a function $\tilde{z}(\cdot) \in H_{0}^{1}\left(I, \mathbb{R}^{N}\right)$ such that $\int_{I} \phi(t, \tilde{z}(t)) d t<l$,

(A4) there is a function $a_{3}(\cdot) \in L^{\infty}(I, \mathbb{R})$ such that

$$
\left|A\left(t, u_{1}\right)-A\left(t, u_{2}\right)\right| \leq a_{3}(t)\left|u_{1}-u_{2}\right|
$$

for each $t \in I$ and each $u_{1}, u_{2} \in U$.

The proof of the theorem on the existence of solutions to (1)-(2) is based on the following version of the well-known Lagrange multiplier rule.

Theorem 1 Ioffe E Tihomirov (1979)Let $z_{*}$ be a local minimum point for the problem

$$
\begin{aligned}
& \mathcal{E}(z) \rightarrow \text { inf }, \\
& g_{i}(z) \leq 0 \quad i=1,2, \ldots, m,
\end{aligned}
$$

where $\mathcal{E}, g_{i}: X \rightarrow \mathbb{R}, X$ is a Banach space. Assume that the functionals $\mathcal{E}$ and $g_{i}, i=1, \ldots, m$ are continuous and Fréchet-differentiable on a neighborhood of $z_{*}$. Then there exist Lagrange multipliers $\lambda_{i} \geq 0, i=0,1, \ldots, m$ not all zero, such that for $i=1,2, \ldots, m$

$$
\begin{aligned}
\lambda_{0} \mathcal{E}^{\prime}\left(z_{*}\right)+\sum_{i=1}^{m} \lambda_{i} g_{i}^{\prime}\left(z_{*}\right) & =0, \\
\lambda_{i} g_{i}\left(z_{*}\right) & =0 .
\end{aligned}
$$

Moreover if there exists a vector $z \in X$ such that

$$
g_{i}^{\prime}\left(z_{*}\right) z<0
$$

for those indices $i=1,2, \ldots, m$, for which $g_{i}\left(z_{*}\right)=0$, then $\lambda_{0} \neq 0$, and it can be assumed without loss of generality that $\lambda_{0}=1$. 
Theorem 2 If assumptions (A1)-(A3) are satisfied then for each each control $u_{0}(\cdot) \in \mathcal{U}$ there are exactly one $\alpha_{0} \geq 0$ and exactly one function $z_{u_{0}}(\cdot) \in H_{0}^{1}\left(I, \mathbb{R}^{N}\right)$ which is a Caratheodory solution to the equation (1) with condition (2).

Proof. Fix $u_{0}(\cdot) \in \mathcal{U}$. Consider the following problem

$$
\begin{aligned}
\mathcal{E}(z(\cdot)) & :=\int_{I}\left(\frac{1}{2}\left|z^{\prime}(t)\right|^{2}+\frac{1}{2}\left\langle A\left(t, u_{0}(t)\right) z(t), z(t)\right\rangle-\left\langle B(t) u_{0}(t), z(t)\right\rangle\right) d t \rightarrow \min , \\
g_{1}(z(\cdot)) & :=\int \phi(t, z(t)) d t-l \leq 0 .
\end{aligned}
$$

Let $\left(z_{n}(\cdot)\right)_{n \in \mathbb{N}}$ be a minimizing sequence for problem (6)-(7), i.e.

$$
\begin{aligned}
& \lim _{n \rightarrow \infty} \mathcal{E}\left(z_{n}(\cdot)\right)=m:=\inf \left\{\mathcal{E}(z(\cdot)): z(\cdot) \in H_{0}^{1}\left(I, \mathbb{R}^{N}\right) \text { and } \int_{I} \phi(t, z(t)) d t \leq l\right\}, \\
& \int_{I} \phi\left(t, z_{n}(t)\right) \leq l .
\end{aligned}
$$

Since $A\left(t, u_{0}(t)\right)$ is positively defined for each $t \in I$ and $A(\cdot, \cdot) \in L^{\infty}\left(I \times U, \mathbb{R}^{N \times N}\right)$, it follows that there exists a function $M(\cdot) \in L^{\infty}(I, \mathbb{R})$ such that

$$
\left\langle A\left(t, u_{0}(t)\right) z(t), z(t)\right\rangle \geq M(t)|z(t)|^{2}, \quad \text { a.e. } t \in I,
$$

for each $z(\cdot) \in H_{0}^{1}\left(I, \mathbb{R}^{N}\right)$, see for example Hestenes (1966). Thus,

$$
\begin{aligned}
& \mathcal{E}\left(z_{n}(\cdot)\right)= \int_{I}\left(\frac{1}{2}\left|z_{n}^{\prime}(t)\right|^{2}+\frac{1}{2}\left\langle A\left(t, u_{0}(t)\right) z_{n}(t), z_{n}(t)\right\rangle-\left\langle B(t) u_{0}(t), z_{n}(t)\right\rangle\right) d t \\
& \geq \int_{I} \frac{1}{2}\left|z_{n}^{\prime}(t)\right|^{2} d t+\int_{I} \frac{1}{2} M(t)\left|z_{n}(t)\right|^{2} d t-\int_{I}\left\langle B(t) u_{0}(t), z_{n}(t)\right\rangle d t \\
& \geq C_{1} \int_{I}\left(\left|z_{n}^{\prime}(t)\right|^{2}+\left|z_{n}(t)\right|^{2}\right) d t-C_{2}\left(\int_{I}\left|z_{n}(t)\right|^{2} d t\right)^{\frac{1}{2}}
\end{aligned}
$$

where $C_{1}>0, C_{2} \in \mathbb{R}$ and both of them do not depend on $n$. The above inequality means that the minimizing sequence $\left(z_{n}(\cdot)\right)_{n \in \mathbb{N}}$ is bounded. The space $H_{0}^{1}\left(I, \mathbb{R}^{N}\right)$ is reflexive. Hence passing if necessary to a subsequence, there exist $z_{*}(\cdot) \in H_{0}^{1}\left(I, \mathbb{R}^{N}\right)$ such that $z_{n}(\cdot) \rightarrow z_{*}(\cdot)$ weakly in $H_{0}^{1}\left(I, \mathbb{R}^{N}\right)$. The functionals $\mathcal{E}$ and $g_{1}$ are convex and continuous, and so weekly lower semicontinuous (see. Mawhin (1987), Mawhin \& Willem (1989)). Therefore

$$
m=\liminf _{n \rightarrow \infty} \mathcal{E}\left(z_{n}\right) \geq \mathcal{E}\left(z_{*}\right)
$$

and

$$
l \geq \liminf _{n \rightarrow \infty} \int_{I} \phi\left(t, z_{n}(t)\right) d t \geq \int_{I} \phi\left(t, z_{*}(t)\right) .
$$

Consequently $z_{*}(\cdot)$ is a minimum point for problem (6)-(7). Moreover, since $\mathcal{E}$ is strictly convex, the minimum point $z_{*}(\cdot)$ is unique. It is easy to check that the functionals $\mathcal{E}$ and $g_{1}$ are Fréchet-differentiable and for each $h(\cdot) \in H_{0}^{1}\left(I, \mathbb{R}^{N}\right)$

$$
\begin{aligned}
& \mathcal{E}^{\prime}(z(\cdot)) h(\cdot)=\int_{I}\left(\left\langle z^{\prime}(t), h^{\prime}(t)\right\rangle+\left\langle A\left(t, u_{0}(t)\right) z(t)-B(t) u_{0}(t), h(t)\right\rangle\right) d t \\
& g_{1}^{\prime}(z(\cdot)) h(\cdot)=\int_{I}\langle\varphi(t, z(t)), h(t)\rangle d t .
\end{aligned}
$$


By (A2), $g_{1}$ is strictly convex, and then

$$
g_{1}(\tilde{z}(\cdot))-g_{1}\left(z_{*}(\cdot)\right)>g_{1}^{\prime}\left(z_{*}(\cdot)\right)\left(\tilde{z}(\cdot)-z_{*}(\cdot)\right) .
$$

for $\tilde{z}(\cdot)$ satisfying $(\mathrm{A} 3)$. Therefore if $g_{1}\left(z_{*}(\cdot)\right)=0$, then

$$
g_{1}^{\prime}\left(z_{*}(\cdot)\right)\left(\tilde{z}(\cdot)-z_{*}(\cdot)\right)<0 .
$$

Now, by the virtue of the Lagrange multiplier rule (Theorem 1 ) we have that there exists $\lambda_{1} \geq 0$ such that

$$
\int_{I}\left\langle z^{\prime}(t), h^{\prime}(t)\right\rangle d t=-\int_{I}\left\langle A(t, u(t)) z(t)-B(t) u(t)+\lambda_{1} \varphi(t, z(t)), h(t)\right\rangle d t
$$

for $h(\cdot) \in H_{0}^{1}\left(I, \mathbb{R}^{N}\right)$. Applying the Du Bois-Reymond Lemma (see Mawhin (1987), Mawhin \& Willem (1989)) we get

$$
-z^{\prime \prime}(t)+A(t, u(t)) z(t)+\alpha_{0} \varphi(t, z(t))=B(t) u(t),
$$

where $\alpha_{0}=\lambda_{1} \geq 0$. The uniqueness of solution follows from the fact that $\mathcal{E}$ and $g_{1}$ are strictly convex.

Theorem 3 Let assumptions (A1)-(A4) are satisfied. Then, for each sequence $\left(u_{k}(\cdot)\right)_{k \in \mathbb{N}}$ of controls tending to $u_{0}(\cdot)$ in $L^{\infty}\left(I, \mathbb{R}^{M}\right)$, the sequence of solutions $z_{k}(\cdot):=z_{u_{k}}(\cdot)$ tends to $z_{0}(\cdot):=z_{u_{k}}(\cdot)$ uniformly on bounded sets and $\lim _{t \rightarrow \infty} z_{0}(t)=0$.

Proof. Consider the sequence of functionals

$$
\mathcal{E}_{k}(z(\cdot)):=\int_{I}\left(\frac{1}{2}\left|z^{\prime}(t)\right|^{2}+\frac{1}{2}\left\langle A\left(t, u_{k}(t)\right) z(t), z(t)\right\rangle-\left\langle B(t) u_{k}(t), z(t)\right\rangle\right) d t .
$$

By (A4), we have that

$$
\begin{aligned}
& \left|\mathcal{E}_{k}(z(\cdot))-\mathcal{E}_{0}(z(\cdot))\right| \\
& \leq \int_{I} \frac{1}{2}\left|A\left(t, u_{k}(t)\right)-A\left(t, u_{0}(t)\right)\right||z(t)|^{2} d t+\int_{I}|B(t)|\left|u_{k}(t)-u_{0}(t)\right||z(t)| d t \\
& \quad \leq \int_{I} a_{3}(t)\left|u_{k}(t)-u_{0}(t)\right||z(t)|^{2} d t+\int_{I}|B(t)|\left|u_{k}(t)-u_{0}(t)\right||z(t)| d t .
\end{aligned}
$$

Since $u_{k}(\cdot)$ tends to $u_{0}(\cdot), \mathcal{E}_{k}$ tends to $\mathcal{E}_{0}$ uniformly on bounded sets, by (8). As $A(t, u(t))$ is positively defined for each $t \in I$ and $\left(A\left(\cdot, u_{k}(\cdot)\right)\right)_{k \in \mathbb{N}}$ is bounded we have that there is a function $M(\cdot) \in L^{\infty}\left(I, \mathbb{R}^{N}\right)$ such that

$$
\left\langle A\left(t, u_{k}(t)\right) z(t), z(t)\right\rangle \geq M(t)|z(t)|^{2}, \quad k \in \mathbb{N} \text { and a.e. } t \in I,
$$

for $z(\cdot) \in H_{0}^{1}\left(I, \mathbb{R}^{N}\right)$. Therefore,

$$
\begin{gathered}
\mathcal{E}_{k}(z(\cdot))=\int_{I}\left(\frac{1}{2}\left|z^{\prime}(t)\right|^{2}+\frac{1}{2}\left\langle A\left(t, u_{k}(t)\right) z(t), z(t)\right\rangle-\left\langle B(t) u_{k}(t), z(t)\right\rangle\right) d t \\
\geq \int_{I} \frac{1}{2}\left|z^{\prime}(t)\right|^{2} d t+\int_{I} \frac{1}{2} M(t)|z(t)|^{2} d t-\int_{I}\left\langle B(t) u_{k}(t), z(t)\right\rangle d t \\
\geq C \int_{I}\left(\left|z^{\prime}(t)\right|^{2}+|z(t)|^{2}\right) d t-\bar{C}\left(\int_{I}|z(t)|^{2} d t\right)^{\frac{1}{2}},
\end{gathered}
$$


for $z(\cdot) \in H_{0}^{1}\left(I, \mathbb{R}^{N}\right)$, where $C$ and $\bar{C}$ do not depend on $k$. Moreover, $\mathcal{E}_{k}(0)=0$ for $k=1,2, \ldots$, thus the set of minimum points for functionals $\mathcal{E}_{k}, k=1,2, \ldots$ is bounded and consequently weakly compact in $H_{0}^{1}\left(I, \mathbb{R}^{N}\right)$. Let $\hat{z}(\cdot) \in H_{0}^{1}\left(I, \mathbb{R}^{N}\right)$ be a cluster point of the above set. Obviously, $\int \phi(t, \hat{z}(t)) d t \leq l$. Suppose that $\hat{z}(\cdot)$ is not a minimum point of $\mathcal{E}_{0}$. Let $z_{0}(\cdot)$ be a minimum point of $\mathcal{E}_{0}$ (such a point exits by Theorem 2 ). Then

$$
\begin{gathered}
\mathcal{E}_{0}\left(z_{0}(\cdot)\right)-\mathcal{E}_{0}(\hat{z}(\cdot))= \\
\left(\mathcal{E}_{0}\left(z_{0}(\cdot)\right)-\mathcal{E}_{k}\left(z_{k}(\cdot)\right)\right)+\left(\mathcal{E}_{k}\left(z_{k}(\cdot)\right)-\mathcal{E}_{0}\left(z_{k}(\cdot)\right)\right)+\left(\mathcal{E}_{0}\left(z_{k}(\cdot)\right)-\mathcal{E}_{0}(\hat{z}(\cdot))\right)
\end{gathered}
$$

and passing with $k \rightarrow \infty$ we have that $\mathcal{E}_{0}(z(\cdot))=\mathcal{E}_{0}(\hat{z}(\cdot))$, since $\mathcal{E}_{k}$ tends to $\mathcal{E}_{0}$ uniformly on bounded sets and $\mathcal{E}_{0}$ is weakly lower semicontinuous.

To complete the proof it is enough to apply the fact that the weak convergence in $H_{0}^{1}\left(I, \mathbb{R}^{N}\right)$ implies the strong convergence in $C_{0}\left(I, \mathbb{R}^{N}\right)$ (cf. Mawhin (1987)) on any bounded set and any minimum point of $\mathcal{E}_{k}$ is a solution and reversely.

If we assume that $A(t, \cdot)$ is linear with respect to $u$ for each $t \in I$, then we can weaken the topology in the set of controls.

In that case we need the following assumption

(A5) the function $A(\cdot, \cdot)$ is of the form

$$
A(t, u)=a(t) u+b(t),
$$

where $a(\cdot) \in L^{\infty}\left(I, \mathbb{R}^{N \times N \times M}\right), b(\cdot) \in L^{\infty}\left(I, \mathbb{R}^{N \times N}\right)$.

Analogously to the proof of Theorem 3 , it is possible to prove

Theorem 4 Suppose (A1)-(A5). Then, for each sequence $\left(u_{k}(\cdot)\right)_{k \in \mathbb{N}}$ of controls tending to $u_{0}(\cdot)$ weakly in $L^{2}\left(I, \mathbb{R}^{M}\right)$, the sequence of solutions $z_{k}(\cdot):=z_{u_{k}}(\cdot)$ tends to $z_{0}(\cdot):=z_{u_{0}}(\cdot)$ weakly in $H_{0}^{1}\left(I, \mathbb{R}^{N}\right)$ and consequently uniformly on bounded sets.

\section{Existence of optimal solution}

In this section we have to assume

(A6) the function $f(\cdot, \cdot, t)$ is convex with respect $(z, u)$ for each $t \in I$,

(A7) there are a constant $b_{1}>0$ and a function $b_{2}(\cdot) \in L^{1}(I, \mathbb{R})$ such that

$$
|f(z, u, t)| \leq b_{1}|z|^{2}+b_{2}(t)
$$

for $z \in \mathbb{R}^{N}, u \in U$ and $t \in I$.

Applying Theorem 4 one can prove that optimal control problem (1)-(5) possesses a solution.

Theorem 5 If control problem (1)-(5) satisfies assumptions (A1)-(A7), then there exist an optimal control in the set of admissible controls $\mathcal{U}(c f$. (5)) . 
Proof. Let $\left(z_{k}(\cdot), u_{k}(\cdot)\right)_{k \in \mathbb{N}} \subset H_{0}^{1}\left(I, \mathbb{R}^{N}\right) \times \mathcal{U}$ be a minimizing sequence for problem (1)(3). Passing, if necessary, to a subsequence we may assume that $\left(u_{k}(\cdot)\right)_{k \in \mathbb{N}}$ tends weakly in $L^{2}\left(I, \mathbb{R}^{M}\right)$ to some $u_{0}(\cdot) \in \mathcal{U}$. By Theorem 4 , the sequence of trajectories $z_{k}(\cdot)$ tends to $z_{0}(\cdot)$ weakly in $H_{0}^{1}\left(I, \mathbb{R}^{N}\right)$. Denote by $\mu$ the optimal value, i.e. $\mu=\inf \{F(x, u)\}$ where infimum is taken over all admissible processes $(z(\cdot), u(\cdot))$ of (1)-(5). Taking into account the lower semicontinuity of the functional $F$ we obtain

$$
\mu=\lim _{k \rightarrow \infty} F\left(z_{k}(\cdot), u_{k}(\cdot)\right) \geq F\left(z_{0}(\cdot), u_{0}(\cdot)\right) \geq \mu .
$$

We thus get $F\left(z_{0}(\cdot), u_{0}(\cdot)\right)=\mu$. It means that the process $\left(z_{0}(\cdot), u_{0}(\cdot)\right)$ is optimal.

\section{Remarks on Schrödinger equation}

The results presented above can be extended to the case of elliptic systems of the form

$$
\triangle z(x)+A(x, u(x)) z(x)+\alpha \varphi(x, z(x))=B(x) u(x), x \in \mathbb{R}^{n}, n \geq 2,
$$

with the integral constraints

and the cost functional

$$
\int_{\mathbb{R}^{n}} \phi(x, z(x)) d x \leq l
$$

$$
\int_{\mathbb{R}^{n}} f(z(x), u(x), x) d x
$$

The set of admissible controls is of the form

$$
\mathcal{U}:=\left\{u(\cdot) \in L^{\infty}\left(\mathbb{R}^{n}, \mathbb{R}^{M}\right): u(x) \in U \subset \mathbb{R}^{M}\right\} .
$$

System (9)-(12) is considered in the Sobolev space $H^{1}\left(\mathbb{R}^{n}, \mathbb{R}^{N}\right)$, see Lieb \& Loss (2001). For system (9)-(12) one can prove theorems analogous to the Theorems 3 - 5. The most interesting is the case, when $n=3, M=N=1, A(x, u)=-u, \varphi(x, z)=z, B=0$ and $\phi(x, z)=\frac{1}{2}|z|^{2}$. In this case system (9) is reduced to the scalar elliptic equation

$$
\triangle z(x)-u(x) z(x)+\alpha z(x)=0,
$$

with the integral condition

$$
\|z\|_{L^{2}\left(\mathbb{R}^{3}, \mathbb{R}\right)}^{2}=\int_{\mathbb{R}^{3}}|z(x)|^{2} d x \leq 1, \text { where } z(\cdot) \in H^{1}\left(\mathbb{R}^{3}, \mathbb{R}\right) .
$$

Equation (13) is the well-known stationary Schrödinger equation, see Lieb \& Loss (2001) and references therein.

From theorem analogous to Theorems 3 - 5, it follows that equation (13) possesses a solution $z_{u}(\cdot) \in H^{1}\left(\mathbb{R}^{3}, \mathbb{R}\right)$ that depends continuously on a potential $u(\cdot) \in L^{\infty}\left(\mathbb{R}^{3}, \mathbb{R}\right)$. Moreover,

$$
\int_{\mathbb{R}^{3}}\left|z_{\mathcal{u}}(x)\right|^{2} d x=1
$$

and

$$
\alpha=\min _{z \in S} \int_{\mathbb{R}^{3}}\left(|\nabla z(x)|^{2}+u(x)|z(x)|^{2}\right) d x,
$$

where $S:=\left\{z \in H^{1}\left(\mathbb{R}^{3}, \mathbb{R}\right): \int_{\mathbb{R}^{3}}\left|z_{\mathcal{u}}(x)\right|^{2} d x=1\right\}$.

The existence of solution to the Schrödinger equation was proved many years ago by applying direct, variational or topological methods. However, as far as we know, the result presented above and concerning continuous dependence of solutions on varying potential $u(x)$ is new. 


\section{Remarks on biological oscillators}

In the work presented above we obtain some results related to the technical or physical applications. Similar methods we can apply to the biological oscillators which are considered, among others, in Murray (2002).

The mathematical models of the oscillators are given by differential systems of the form

$$
\begin{aligned}
& \frac{d z^{1}(t)}{d t}=f\left(z^{n}(t)\right)-k_{1} z^{1}(t) \\
& \frac{d z^{r}(t)}{d t}=z^{r-1}(t)-k_{r} z^{r}(t), \quad r=2,3, \ldots, n
\end{aligned}
$$

where $f$ is a continuous function, $k_{i}>0, i=1,2, \ldots, n$.

System (15) is investigated with the Cauchy initial conditions or the Dirichlet boundary conditions.

In particular cases system (15) may be reduced to the scalar differential equation

$$
v^{(n)}(t)+a_{n-2} v^{(n-2)}(t)+\ldots+a_{2} v^{\prime \prime}(t)=\varphi(t, v(t))
$$

where $n$ is an even natural number, $a_{i} \in \mathbb{R}, \varphi(t, \cdot)$ is a continuous function. Systems of this form are often referred to as the Newton system of the $n$-th order. (If $n=2$, we have classical Newton system $a_{2} v^{\prime \prime}(t)=\varphi(t, v(t))$ with $\left.a_{2} \neq 0\right)$. The differential equation of the form (16) can be investigated by means of variational methods, in a similar way to equation (1).

\section{Remarks on systems with variable mass}

The variational method presented in the paper might be applied to the investigation of systems with variable mass. Let us consider an object $Q$ with $k \geq 1$ engines $I_{s}$ that emit gases with velocities $v_{s}=v_{s}(t), s=1,2, \ldots, k ; t \in[0, T]$ for a fixed $T>0$. For example $Q$ might be an airplane, a rocket and the like, see for example Meriam \& Kraige (2002).

A motion of the object $Q$ in $\mathbb{R}^{n}$ may be described by the equation

$$
m(t) \frac{d v(t)}{d t}=\sum_{s=1}^{k}\left(v_{\mathcal{S}}(t)-v(t)\right) \frac{d m_{S}(t)}{d t}+f(t, x(t), u(t))
$$

where $m(t)$ is the mass of the object $Q$ at time $t \in[0, T] ; v_{S}(t)$ stands for the velocity of the emitted gas by the engine $I_{s} ; v(t)$ denotes the velocity of the object $Q ; m_{S}(t)$ is the mass of the engine $I_{s} ; f$ denotes an external force (for example the gravity force) acting on the object $Q$ that may depend on the location of object $x(t)=x(0)+\int_{0}^{t} v(\tau) d \tau$, and finally $u(t)$ is a control.

The equation (17) is so called the Meščerskii equation and appears in mathematical models of systems with variable mass (see Kosmodemianskii (1966), Peraire (2002), Meščerskii (1962)). In a particular case, if we assume that $f=0, k=1$ and $v_{1}(t)-v(t)=$ const, then the equation (17) reduces to the Tsiolkovskii equation of the form

$$
m(t) \frac{d v(t)}{d t}=c \frac{d m(t)}{d t} .
$$

Integrating the equation (18), we obtain

$$
v(t)-v(0)=c \ln \frac{m(t)}{m(0)} .
$$


The equation (19) describes the dependence of the increase in the velocity of the object $Q$ and the loss in its mass. In the same way we can interpret a solution to the equation (17). From the equation (19) it follows that the increase in velocity is a nonlinear function of mass. If $v(t)=x^{\prime}(t)$ and $v_{S}(t)-v(t)=c(t)$, then the equation (17) has the form

$$
x^{\prime \prime}(t)=F(t, x(t), \mu(t), u(t))
$$

where $x(t)$ stands for the location of the object $Q$ at time $t \in[0, T], \mu=\left(\mu_{1}, \mu_{2}, \ldots, \mu_{k}\right)$ with $\mu_{s}(t)=\frac{d m_{s}(t)}{d t}, s=1,2, \ldots, k, u(\cdot)$ is some external control and the function $F$ is given by

$$
F(t, x, \mu, u)=\frac{1}{m(t)}\left[\sum_{s=1}^{k} c_{S}(t) \mu_{s}(t)+f(t, x(t), u(t))\right] .
$$

Let us assume that a relative velocity of the emitted gas is a given function $c_{S}(t)=v_{S}(t)-v(t)$ for any $s=1,2, \ldots, k$ and $t \in[0, T]$. Moreover, for any $s=1,2, \ldots, k$, the function $\mu_{s}$ controls the power of the engine $I_{S}$ on the interval $[0, T]$.

The equation (20) may be considered with the boundary conditions of the Dirichlet type

$$
x(0)=x_{0}, x(T)=x_{T},
$$

and with the constrains on controls

$$
\begin{aligned}
& \mu(\cdot) \in \mathcal{M}=\left\{\mu(\cdot) \in L^{\infty}\left([0, T], \mathbb{R}^{k}\right) ; \mu(t) \in M \subset \mathbb{R}^{k}\right\}, \\
& u(\cdot) \in \mathcal{U}=\left\{u(\cdot) \in L^{\infty}\left([0, T], \mathbb{R}^{m}\right) ; \mu(t) \in U \subset \mathbb{R}^{m}\right\}
\end{aligned}
$$

and state coordinates

$$
\int_{0}^{T} \varphi(t, x(t)) d t \leq l
$$

Let us consider the cost functional of the form

$$
G(x, \mu, u)=\int_{0}^{T} g(t, x(t), \mu(t), u(t)) d t
$$

We impose the following assumptions:

(M1) the function $f:[0, T] \times \mathbb{R}^{n} \times \mathbb{R}^{m} \rightarrow \mathbb{R}^{n}$ is continuous and linear with respect to $u$,

(M2) the function $\varphi:[0, T] \times \mathbb{R}^{n} \rightarrow \mathbb{R}$ is continuous and convex with respect to $x$,

(M3) the integrand $g:[0, T] \times \mathbb{R}^{n} \times \mathbb{R}^{k} \times \mathbb{R}^{m} \rightarrow \mathbb{R}$ is continuous and convex with respect to $(\mu, u)$.

Applying the method presented in the first section of the chapter we can prove the theorem on the existence of optimal processes to system (20) - (24) that is analogous to Theorem 5. 


\section{References}

Adams, R. A. (1975). Sobolev spaces, Academic Press, New York.

Hestenes, M. R. (1966). Calculus of Variations and Optimal Control Theory, John Wiley and Sons, New York.

Ioffe, A. D. \& Tihomirov, V. M. (1979). Theory of Extremal Problems, North-Holland Publishing Company, Amsterdam, New York, Oxford.

Kufner, A., John, O. \& Fučik, S. (1977). Function spaces, Academia, Prague, and Noordhoff, Leyden.

A.A. Kosmodemianskii, A. A. (1966). The course of theoretical mechanics, Moscow (in Russian).

Lieb, E. H. \& Loss, M. (2001). Analysis, AMS, 2001.

Łojasiewicz, S. (1974). An Introduction to the Theory of Real Functions, John Willey and Sons Ltd., Chichester.

Mawhin, J. (1987). Problémes de Dirichlet Variationnels Non-Linéaires. Les Presses de L'Universté de Montréal, Canada.

Mawhin, J. \& Willem, M. (1989). Critical Point Theory and Hamiltonian Systems, Springer-Verlag, New York.

Meriam J. L. \& Kraige, L. G. (2002). Engineering mechanics, Dynamics,5th edition, John Wiley \& Sons.

Meščerskii I. V. (1962). Works on mechanics of the variable mass, Moscow (in Russian).

Murray, J. D. (2002). An Introduction to Mathematical Biology, Springer-Verlag.

Peraire, J. (2004). Variable mass systems: the rocket equation, MIT OpenSourceWare, Massachusetts Institute of Technology, available online. 


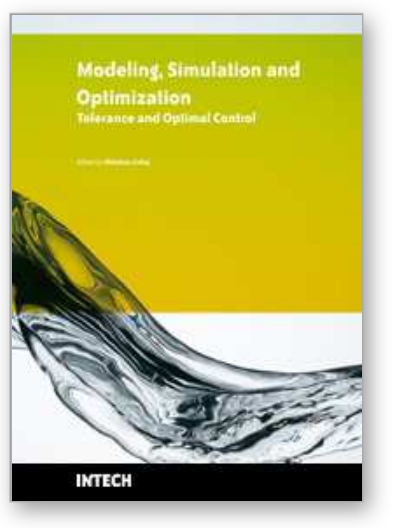

\section{Modeling Simulation and Optimization - Tolerance and Optimal Control}

Edited by Shkelzen Cakaj

ISBN 978-953-307-056-8

Hard cover, 304 pages

Publisher InTech

Published online 01, April, 2010

Published in print edition April, 2010

Parametric representation of shapes, mechanical components modeling with 3D visualization techniques using object oriented programming, the well known golden ratio application on vertical and horizontal displacement investigations of the ground surface, spatial modeling and simulating of dynamic continuous fluid flow process, simulation model for waste-water treatment, an interaction of tilt and illumination conditions at flight simulation and errors in taxiing performance, plant layout optimal plot plan, atmospheric modeling for weather prediction, a stochastic search method that explores the solutions for hill climbing process, cellular automata simulations, thyristor switching characteristics simulation, and simulation framework toward bandwidth quantization and measurement, are all topics with appropriate results from different research backgrounds focused on tolerance analysis and optimal control provided in this book.

\section{How to reference}

In order to correctly reference this scholarly work, feel free to copy and paste the following:

Dorota Bors, Marek Majewski and Stanislaw Walczak (2010). Optimal Control Systems with Constrains Defined on Unbounded Sets, Modeling Simulation and Optimization - Tolerance and Optimal Control, Shkelzen Cakaj (Ed.), ISBN: 978-953-307-056-8, InTech, Available from: http://www.intechopen.com/books/modelingsimulation-and-optimization-tolerance-and-optimal-control/optimal-control-systems-with-constrains-defined-onunbounded-sets

\section{INTECH}

open science | open minds

\section{InTech Europe}

University Campus STeP Ri

Slavka Krautzeka 83/A

51000 Rijeka, Croatia

Phone: +385 (51) 770447

Fax: +385 (51) 686166

www.intechopen.com

\section{InTech China}

Unit 405, Office Block, Hotel Equatorial Shanghai

No.65, Yan An Road (West), Shanghai, 200040, China

中国上海市延安西路65号上海国际贵都大饭店办公楼 405 单元

Phone: +86-21-62489820

Fax: $+86-21-62489821$ 
(C) 2010 The Author(s). Licensee IntechOpen. This chapter is distributed under the terms of the Creative Commons Attribution-NonCommercialShareAlike-3.0 License, which permits use, distribution and reproduction for non-commercial purposes, provided the original is properly cited and derivative works building on this content are distributed under the same license. 